\title{
ÍNDICE DE PRIORIZAÇÃO TECNOLÓGICA DAS COMUNIDADES QUILOMBOLAS (IPTe_Qsc) - Estado da Paraíba
}

\author{
Angela Carolina de MEDEIROS ${ }^{1}$, Tatiana Ayako TAURA ${ }^{2}$, Aderaldo de Souza SILVA ${ }^{3}$ \\ ${ }^{1}$ Dra. Bióloga, Salvaguardas Socioambientais, Projeto Cooperar-PB/BIRD - BR 230 - Km 14 - S/N - Cabedelo/PB - CEP \\ 58.310-000 - Telefones: PABX (83) 3246-8644 - FAX (83) 3246-7858, angelacarolinademedeiros@ gmail.com \\ 2 Msc. Eng. Cartógrafa, Analista, Embrapa Semiárido, BR 428-km 152 - Zona Rural - C.P. 23, Fone: (87) 3866.3803, CEP \\ 56302-970. Petrolina-PE, tatiana.taura@embrapa.br \\ ${ }^{3}$ Dr. Eng. Agrônomo, pesquisador, Embrapa Semiárido, BR 428-km 152 - Zona Rural - C.P. 23, Fone: (87) 3866.3803, CEP \\ 56302-970. Petrolina-PE, aderaldo.silva@embrapa.br
}

RESUMO: O estudo teve como objetivo a construção do Índice de Priorização Tecnológica (IPTe_Qsc), que possibilitou a geoespacialização das tecnologias de Convivência com o Semiárido para 38 comunidades quilombolas do Estado da Paraíba. O algoritmo estatístico associado ao Sistema de Informação Geográfica, por setor censitário, permitiu definir as prioridades tecnológicas socioambientais fundamental, primária, secundária e terciária para esta população. A pesquisa comprovou que as comunidades localizadas nos setores censitários da Zona Rural, são as mais vulneráveis à priorização tecnológica que as de Área Urbanizada de cidade ou vila. A Comunidade Quilombola de Vila Teimosa, pertencente ao município de Livramento foi considerada a mais vulnerável. O IPTe_Qsc sugeriu priorizá-la com: agricultura irrigada, áreas de preservação e/ou conservação ambiental, construção de barreiros para uso com irrigação de salvação e/ou pequenas barragens de terra, exploração das terras agricultáveis com técnicas de captação de água de chuva in situ e turismo. As demais comunidades foram agrupadas em classes de vulnerabilidade: alta (3), média (21) e baixa (13), seguindo-se técnicas, métodos e procedimentos similares.

PALAVRAS-CHAVE: geoespacialização, políticas públicas, vulnerabilidade.

\section{TECHNOLOGICAL PRIORIZATION INDEX OF THE QUILOMBOLAS COMMUNITIES (IPTe_Qsc) - State of Paraiba}

SUMMARY: The objective of this study was the construction of the Technological Prioritization Index (IPTe_Qsc), which enabled the geospatial technology of the Coexistence with the Semi-Arid to 38 communities in the state of Paraíba. The statistical algorithm associated with the Geographic Information System, by census tract, allowed defining the socio-environmental technological priorities fundamental, primary, secondary and tertiary for this population. The research proved that the communities located in the census tracts of the Rural Zone are the most vulnerable to the technological prioritization of those in Urbanized Area of city or town. The Quilombola Community of Vila Teimosa, belonging to the municipality of Livramento was considered the most vulnerable. The IPTe_Qsc suggested to prioritize it with: irrigated agriculture, areas of preservation and / or environmental conservation, construction of barriers for use with irrigation and / or small earth dams, exploitation of arable land using rainwater harvesting techniques in Situ and tourism. The other communities were grouped into vulnerability classes: high (3), medium (21) and low (13), followed by similar techniques, methods and procedures

KEYWORDS: Geostatistics, public policies, vulnerability 


\section{INTRODUÇÃO}

O estudo das 38 Comunidades Quilombolas do Estado da Paraíba foi consolidado por meio da construção do Índice de Priorização Tecnológica (IPTe_Qsc), o qual requereu conhecimento nas áreas de cartografia e Sistema de Informações Geográficas, orientados para o desenvolvimento de métodos e técnicas de levantamento, processamento e análise de dados georreferenciados, no âmbito dos Setores Censitários do Instituto Brasileiro de Geografia e Estatística (IBGE), tanto no espaço bidimensional como tridimensional, para a criação de seu algoritmo de análise multidimensional. Neste contexto, o IPTe_Qsc proporciona o acervo tecnológico georreferenciado por setor censitário, onde encontram-se localizadas as comunidades avaliadas.

Assim, o objetivo principal deste trabalho consiste em classificar hierarquicamente o acervo tecnológico georreferenciado atual para cada comunidade quilombola estudada a partir do IPTe_Qsc.

\section{MATERIAL E MÉTODOS}

Foi criado o banco de dados para a construção do IPTe_Qsc com informações provenientes das potencialidades socioeconomicas da Paraíba (FIEP, 2009), informações do censo quilombola (AACADE, 2014), análises físico-química e microbiológica das águas in loco, informações do Censo Demográfico do IBGE (IBGE, 2010) e Zoneamento Agroecológico do Nordeste (ZANE, 2002).

As principais tecnologias, georreferenciadas e codificadas foram as seguintes: Avicultura (E1); Fruticultura dependente de chuva (E2); Cana de açúcar (Capineira) (E3); Côco da bahia (E4); Floricultura (E5); Abastecimento e Saneamento Básico (G1); Agricultura irrigada (G2); Agricultura, horticultura ecológica (G3); Oleaginosas (G4); Apicultura (G6); Área preservação, conservação Ambiental (G7); Barragem subterrânea (G8); Barreiro de salvação (Pequenas Barragens de Terra) (G9); Caprinocultura/Ovinocultura (T1); Captação in situ (T2); Fruticultura irrigada (T3); Irrigação de salvação, complementar (T4); Piscicultura (T5); Turismo (Radical, rural, cultural, etc) e/ou Hotel Fazenda (T7); Minério não metálico e metálico (T8); Forrageiras e capineiras (T9).

Além disso, foram georreferenciadas e codificadas as tecnologias citadas por técnicos pesquisadores da Associação de Apoio aos Assentamentos e Comunidades Afrodescendentes da Paraíba (AACADE), provenientes de demandas in loco, tais como: Construção de cisterna para armazenamento de água de chuva (S1); Tratamento caseiro e/ou comunitário de água residuária (S2); Reuso de água (S3); Tecnologia de geração de renda familiar (Sisteminha 
Embrapa) (S4); Criação de Cooperativa de Pró-Catadores (S5); Coleta seletiva de materiais recicláveis (S6); Eliminação ou construção de Aterro sanitário comunitário (S7); Centro Comunitário de Capacitação e Multi-Negócios (S8); Perfuração de poço profundo (S9); Construção e/ou manutenção de Sistema simplificado de abastecimento de água (U1); Recuperação de Sistema de Saneamento Básico (U2); Reforma e construção de casa (U3); Mobilidade rural (Construção de passagem molhada, estrada vicinal, reforma e/ou construção de açudes de usos múltiplos) (U4); Reforma e/ou ampliação de escola (U5); e Redução da desertificação severa de terras agricultáveis (T6).

\section{RESULTADOS E DISCUSSÃO}

A combinação linear das 30 variáveis utilizadas na análise fatorial explicou a correlação para o conjunto das 38 comunidades avaliadas. Como resultado foram definidas quatro macro características, cujos valores das comunalidades finais foram superiores a 45,46\%, considerados significativos $(\mathrm{P} \leq 0,001)$. Essas características conceituais foram denominadas perfis das comunidades: $1^{\circ}$ ) comunidades urbanizadas de cidade; $2^{\circ}$ ) extrema pobreza; $3^{\circ}$ ) risco climático; e $4^{\circ}$ ) agricultura familiar.

A Tabela 1 e a Figura 1 apresentam a síntese das análises multidimensionais, contemplando os resultados provenientes da matriz de coeficientes (perfis 3, 1, 2 e 4) e da matriz de tipificação da vulnerabilidade (classes 1, 2, 3 e 4), nesta ordem sequencial. Além disso, a Tabela 1, também, apresenta valores médios para as variáveis significativas em função das classes de vulnerabilidade: elevada, alta, média e baixa. A vulnerabilidade das classes foi definida pela variável discriminante referente ao Número de Domicílios Particulares com Rendimentos Nominais Mensais Domiciliares per capita de até 1/8 de Salário Mínimo, altamente significativa para a Classe 1, totalizando valores médios de 102 domicílios, em relação as classes alta, média e baixa, que apresentaram valores médios de 40, 33 e 31 , respectivamente.

A distribuição hierárquica de cada comunidade, seu respectivo índice IPTe_Qsc e as geotecnologias, também, podem ser observados na Tabela 1.

Tabela 1. Índice de Priorização Tecnológica das Comunidades Quilombolas (IPTe_Qsc) do Estado da Paraíba por setor censitário do IBGE.

\begin{tabular}{cccclcc}
\hline ND & P & Setor Censitário & \multicolumn{1}{c}{ Comunidades } & \multicolumn{1}{c}{ Geotecnologias - Codificadas } & C & IPTe \\
\hline 1 & 3 & 250850505000007 & Vila Teimosa & E3G2G7G9T2T7U3 & $\mathbf{1}$ & 0.0464 \\
2 & 1 & 250480105000019 & Negra de Santa Tereza & G7T2S2S3S4S7S8U1 & $\mathbf{2}$ & 0.0460 \\
3 & 1 & 251340605000014 & Quilombo Urbano & S2S3S6U3 & G4G6T1T2S2S3S5S6S8 & 0.0453 \\
4 & 1 & 251210105000011 & Daniel Urbano & $\mathbf{2}$ & 0.0445
\end{tabular}




\begin{tabular}{|c|c|c|c|c|c|c|}
\hline 5 & 2 & 250850505000006 & Sussuarana & E3E5G2G6G7G9T2T7S1S8S9U2U3 & 3 & 0.0438 \\
\hline 6 & 2 & 250355505000011 & Serra Feia & E3E5G2G6G9T2T7S6S7S8U2U3U4 & 3 & 0.0426 \\
\hline 7 & 2 & 250570905000006 & Cruz da Menina & E4T1T2S6S7S8U2U3 & 3 & 0.0415 \\
\hline 8 & 2 & 250900805000007 & Fonseca & E3G2G6G7G9T2T3T7S8U2U3 & 3 & 0.0403 \\
\hline 9 & 2 & 250375305000003 & Umburaninha & E3G2G6G7G9T2T3T7T8S8U1U2U3 & 3 & 0.0392 \\
\hline 10 & 2 & 250940420000006 & Matão & E1E3E4G2T3T7S8S9 & 3 & 0.0381 \\
\hline 11 & 2 & 251280405000005 & Contendas & E1E3E4G2G8T3T7S8S9U1U3U4 & 3 & 0.0369 \\
\hline 12 & 2 & 251275405000005 & Grilo & E1E3E4G2T3T7S1S8U3 & 3 & 0.0358 \\
\hline 13 & 2 & 250355505000010 & Aracati/Chã & G4G6G8T1T2T3 & 3 & 0.0346 \\
\hline 14 & 2 & 250430605000021 & Pau de Leite & G4G6G8T1T2T3S1S6S7S8U1U4 & 3 & 0.0335 \\
\hline 15 & 2 & 250560005000004 & Sitio Vaca Morta & G4G6G7G8T1T2T3S8S9 & 3 & 0.0323 \\
\hline 16 & 2 & 250375305000002 & Vinhas & G4G6G7G8T1T2S4U1U3 & 3 & 0.0312 \\
\hline 17 & 2 & 250430605000023 & São Pedro & E1E3E4T3T7S4S8S9U2U3 & 3 & 0.0301 \\
\hline 18 & 2 & 250430610000005 & Curralinho/Jatobá & E4T1T2T3S4S8S9U2U3 & 3 & 0.0289 \\
\hline 19 & 2 & 251710005000004 & Pitombeira & G7G8T2T3T7S7S8U1 & 3 & 0.0278 \\
\hline 20 & 2 & 251210105000032 & Rufino do Sitio São João & G2G4T3T7T8S8S9U1U2U3U4 & 3 & 0.0266 \\
\hline 21 & 2 & 250430625000002 & Lagoa Rasa & G7T2T3T7S8S9 & 3 & 0.0255 \\
\hline 22 & 2 & 251580705000008 & Negra de Sitio Matias & G4G8T3T7S1S8U2U3U4U5 & 3 & 0.0244 \\
\hline 23 & 2 & 251490905000011 & Serra do Talhado & G6G7T1T2T8S1S9U4 & 3 & 0.0232 \\
\hline 24 & 2 & 251140005000028 & Serra do Abreu & G4G8G9T1S1S6S7S8S9U2U3 & 3 & 0.0221 \\
\hline 25 & 2 & 251340605000020 & Areia de verão & T1T7S1S6S8S9U2U3U4 & 3 & 0.0209 \\
\hline 26 & 4 & 250680607000003 & Pedra D'agua & G7T2S1S8S9U2U3U4U5 & 4 & 0.0198 \\
\hline 27 & 4 & 250560005000005 & Barra de Oitis & G4G6G8T1T3S1S4S8S9U2U3 & 4 & 0.0183 \\
\hline 28 & 4 & 250460305000012 & Gurugi & E3G2G6G7G9T2T3T7 & 4 & 0.0167 \\
\hline 29 & 4 & 250480105000012 & Negra de Barreiras & E3G2G6G7G9T2T3T7T8S4U4 & 4 & 0.0152 \\
\hline 30 & 4 & 250750705000961 & Negra do Paratibe & G4G6G8T1 & 4 & 0.0137 \\
\hline 31 & 4 & 250030405000032 & Caiana dos Criolos & G6G7T1T7S1U1U3U4 & 4 & 0.0122 \\
\hline 32 & 4 & 251660705000025 & Domingos Ferreira & G2G7S1S6S7S8U1U3U4 & 4 & 0.0107 \\
\hline 33 & 4 & 250460305000033 & Ipiranga & G7T2T3T7T8S9U1U3U5 & 4 & 0.0091 \\
\hline 34 & 4 & 250480105000013 & Negra de Mãe D'agua & E4T1T2T3 & 4 & 0.0076 \\
\hline 35 & 4 & 251455205000006 & Sitio Livramento & T1S8S9U3U4 & 4 & 0.0061 \\
\hline 36 & 4 & 250110415000003 & Engenho Novo & E4G4G7G8T2T3S8U2U3 & 4 & 0.0046 \\
\hline 37 & 4 & 250110410000005 & Engenho do Bonfim & G7T2T3T7T8S1S8U2U3U4 & 4 & 0.0030 \\
\hline 38 & 4 & 250460305000034 & Mituaçu & G2G7T7T8S8U4 & 4 & 0.0015 \\
\hline
\end{tabular}

NOTA: ND - Número de Ordem; P - Perfis da Comunidade; C - Classe; IPTe - Índice de Priorização Tecnológica; e GEO- Tecnologias de conivência com o Semiárido, geoespacializadas e codificadas.

Classe 1 - Vulnerabilidade Elevada (cor vermelha) - as caracteristicas das comunidades quilombolas agrupadas nessa classe foram definidas pelo perfil 3 (risco climático). Esse perfil foi caracterizado pelas variáveis: precipitação pluviométrica média anual, tipo de clima predominante, mês representativo do término do período chuvoso e índice climático do setor censitário onde a comunidade encontra-se localizada. As estimativas das comunalidades finais corroboraram com explicação de 98,64\%, 65,33\%, 64,46\% e 63,88\%, respectivamente. 
O IPTe_Qsc foi $\leq$ a 0,0464 e agrupou, exclusivamente, a comunidade quilombola de Vila Teimosa, pertencente ao município de Livramento, na região do médio Sertão. Essa comunidade apresentou potencialidade para inserção de geotecnologias relacionadas a: Exploração de capineiras com Cana-de-açúcar (E3); Agricultura irrigada (G2); Áreas de preservação e/ou conservação ambiental (G7); Construção de barreiros para uso com irrigação de salvação e/ou pequenas barragens de terra (G9); Exploração das terras agricultáveis com técnicas de captação de água de chuva in situ (T2); Turismo (T7); e Demandas para construção e reformas de moradias (U3).

Classe 2 - Vulnerabilidade Alta (cor amarela), representada pela variação do IPTe_Qsc entre 0,0445 e 0,0460, agrupa as comunidades de Negra de Santa Tereza (Coremas), Quilombo Urbano (Santa Luzia) e Daniel Urbano (Pombal), nos setores censitários pertencentes ao Perfil 1, denominado de Comunidades Urbanizadas de cidade ou vila, segundo o IBGE (2010).

Em relação às geotecnologias, destacam-se: tratamento de água de consumo in natura, inclusive residuária (S2); reuso de água (S3); criação de cooperativa de pró-catadores (S5); coleta seletiva (S6); e aterro sanitário comunitário sustentável (S7).

Salienta-se que na pesquisa da Associação de Apoio aos Assentamentos e Comunidade Afrodescendentes (AACADE), um segmento importante das famílias das comunidades da Classe 2, declarou que não realizava tratamento das águas provenientes de fontes tradicionais usadas para consumo humano. Entretanto, quando questionadas se a água para consumo in natura recebia algum tipo de tratamento, afirmaram que sim. $\mathrm{O}$ tratamento era com hipoclorito de cloro cedido pelos agentes de saúde locais.

Classe 3 - Vulnerabilidade Média (cor verde), agrupou 21 comunidades quilombolas pertencentes ao Perfil 2 (extrema pobreza), com IPTe_Qsc entre $0.0209 \geq$ a 0.0438,

As geotecnologias indicadas para as comunidades dessa Classe foram diversificadas, destacando-se as potencialidades para: avicultura (E1), barragens subterrâneas (G8), poços profundos (S9), e demanda por centro comunitário, todas voltadas à capacitação empreendedora e aos multi-negócios das famílias quilombolas, sem paternalismo (S8).

Classe 4 - Vulnerabilidade baixa (cor azul), cujos intervalos de IPTe_Qsc tem valores $\leq$ a 0,0015 ou $\geq$ a 0.0193 . Nessa Classe foram agrupadas 13 comunidades cujas atividades foram definidas pelo Perfil 4 (Agricultura Familiar). Salienta-se que a maioria dessas famílias foram beneficiárias do Programa Hum milhão de cisternas rurais, do Governo Federal.

Para essa Classe destacam-se as geotecnologias: fruticultura irrigada (T3), Agricultura, horticultura ecológica (G3) e piscicultura (T5). 


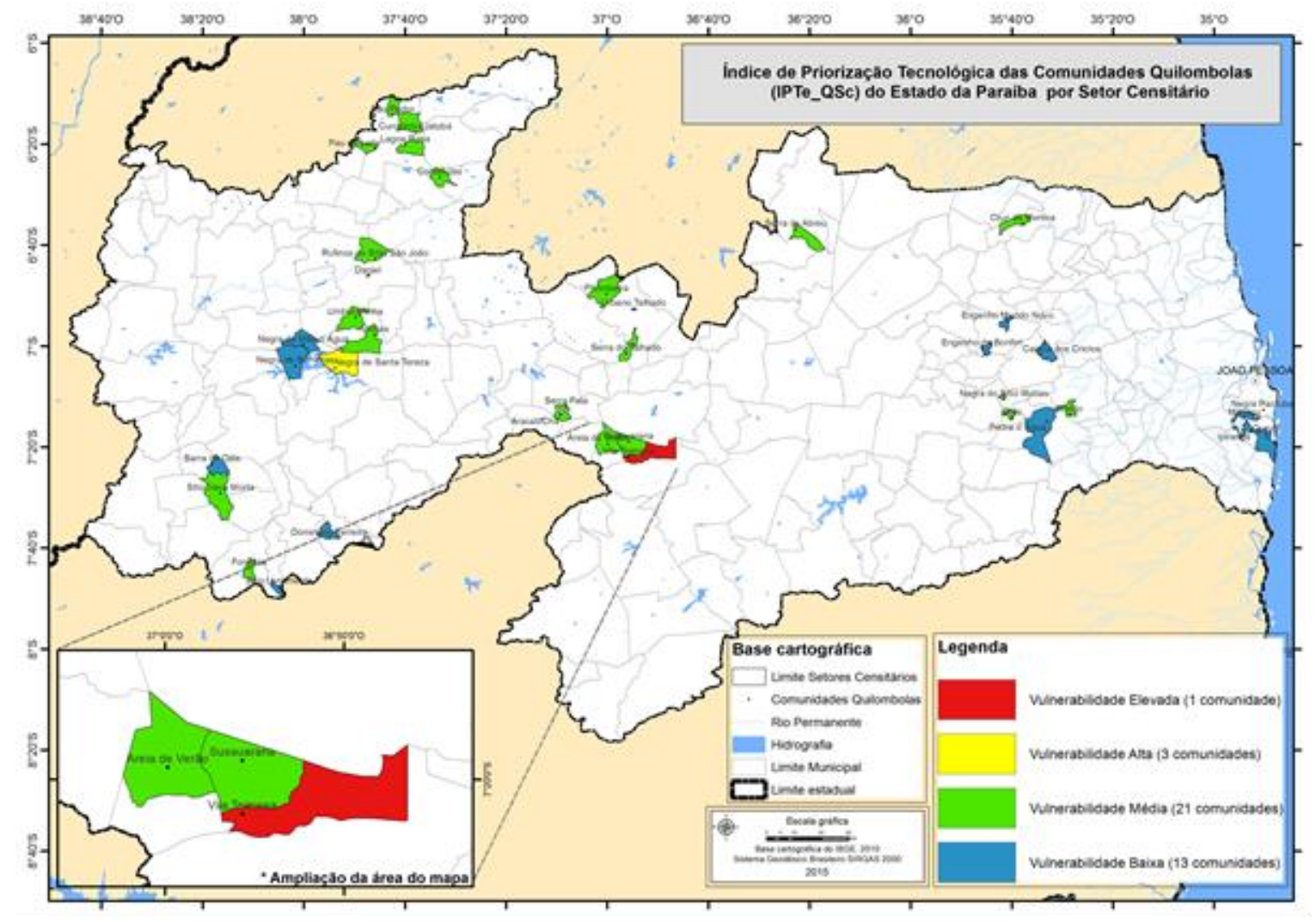

Figura 1 - Mapa do Índice de Priorização Tecnológica das Comunidades quilombolas (IPTe_QSc) do Estado da Paraíba.

\section{CONCLUSÕES}

A pesquisa comprovou que as comunidades localizadas nos setores censitários da Zona Rural, são as mais vulneráveis à priorização tecnológica do que as de Área Urbanizada de cidade ou vila. A partir da construção do Índice de Priorização Tecnológica (IPTe_Qsc) foi possível determinar as causas fundamental, primária, secundária e terciária das 38 comunidades do Estado da Paraíba com a finalidade de elencar políticas públicas socioambientais em base sustentável para esta população.

\section{REFERÊNCIAS}

AACADE. Associação de Apoio aos Assentamentos e Comunidade Afrodescendentes. Estudo Censitário da População Quilombola da Paraíba. João Pessoa, 2014.

FIEP - Federação das Indústrias do Estado da Paraíba. Mapa de Oportunidades do Estado da Paraíba. Áreas Potenciais de Investimento. Campina Grande, 2009.

IBGE - Instituto Brasileiro de Geografia e Estatística. Censo 2010. Brasília/DF, 2010.

ZANE. Zoneamento Agroecológico do nordeste do Brasil: diagnóstico e prognóstico. Embrapa Solos: Recife, 2002. 